\title{
Variation outside variable segments of the major outer membrane protein distinguishes trachoma from urogenital isolates of the same serovar of Chlamydia trachomatis
}

\author{
E H Frost, S Deslandes, D Gendron, D Bourgaux-Ramoisy, P Bourgaux
}

\begin{abstract}
Objectives-Whereas serovars A, B, Ba and $C$ of Chlamydia trachomatis are usually associated with trachoma, two of these serovars (Ba and $\mathrm{C}$ ) are occasionally observed in urogenital infections. Variation in the gene encoding the major outer membrane protein (MOMP) was explored to distinguish urogenital from trachoma specimens of the same serovar. Methods-A large portion of the MOMP gene was amplified by nested PCR directly from clinical samples from trachoma or urogenital infection and the serovar of the infecting $C$ trachomatis was determined by restriction fragment length polymorphism (RFLP). Amplified DNA from trachoma serovars $B, B a$ and $C$ and from urogenital serovars $B a, C, D$ and $E$ was sequenced by the dideoxy chain termination method.
\end{abstract}

Results-While almost identical in variable segment (VS)I, three urogenital Ba samples differed from all trachoma $B$ and $\mathrm{Ba}$ samples at eight nucleotides including two sites which changed amino acids in the constant region upstream of VSI. An identical sequence in this region was observed for the reference urogenital D serovar. Variation in this same region upstream of VSI also distinguished $40 \%$ of serovar D samples from prototype $D$ including three that were sequenced. Two urogenital $C$ differed from trachoma $C$ samples at four sites that changed the MOMP amino acid sequence including two changes in the constant region between VSII and III and single changes in VSII and III. On the basis of these sequence determinations, RFLP was predicted which allowed extension of these observations to 20 other urogenital $\mathrm{Ba}, 12$ trachoma $B$ or $B a$, seven variant $D, 12 D$, four urogenital $C$ and three trachoma $C$ samples without further sequencing.

Conclusion-Urogenital $\mathrm{Ba}$ and $\mathrm{C}$ samples have VSI or II and III sequences identical or very similar to trachoma strains of the same serovar, but resemble more closely other serovars in the constant regions. Urogenital serovar D samples can also be divided into two genotypes on the basis of sequence differences in the constant region preceding VSI.

(Genitourin Med 1995;71:18-23)
Keywords: Chlamydia trachomatis; trachoma; major outer membrane protein

\section{Introduction}

Chlamydia trachomatis is a major world health problem not only as a sexually-transmitted pathogen, but also as the causal agent of trachoma. Although $C$ trachomatis isolates can be distinguished by a serological typing scheme based on variation in the major outer membrane protein (MOMP), there is no clear distinction between the serovars causing trachoma from those associated with cervicitis and urethritis. For example, the serovar C associated with trachoma resembles more closely the urogenital serovar $\mathrm{J}$ than the other trachoma types A, B and Ba. Furthermore, several studies have noted a small percentage of $\mathrm{Ba}$ and $\mathrm{C}$ serovars (reviewed in refs 1,2 ) among urogenital specimens.

The serological typing procedure detects surface-exposed epitopes situated in four welldefined regions of the MOMP, termed variable segments (VS). Sequencing the entire MOMP gene from several serovars ${ }^{3}$ and cataloguing the sequences that correspond to the VS of all 15 serovars ${ }^{4}$ has confirmed the molecular basis of the serological typing procedure and provided an alternative method for distinguishing serovars either by direct sequencing of the VS ${ }^{15}$ or by restriction fragment length polymorphism (RFLP) (reviewed in 2) in PCR-amplified MOMP gene sequences. Direct sequencing has highlighted heterogeneity within the VS from both trachoma ${ }^{56}$ and urogenital specimens. ${ }^{17}$ Recently VSI, II and IV of five urogenital $\mathrm{Ba}$ and one urogenital C samples were sequenced. ${ }^{7}$ Although the urogenital $\mathrm{Ba}$ samples all resembled previously sequenced $\mathrm{Ba}$ variants from trachoma, the urogenital $\mathrm{C}$ sample differed from the trachoma reference $C$ serovar at multiple sites in the VS. ${ }^{7}$ RFLP studies have identified variation outside the VS. ${ }^{28}$ Although heterogeneity outside the VS probably would not influence classification by a serologically-based typing scheme, it could influence cellular tropism, biovar specificity or virulence.

Materials and methods

Chlamydiae. Reference strains B-TW-5/OT, C-TW-3/OT, D-UW-3/Cx, and E-UW-5/Cx were supplied by the Quebec Public Health Laboratory (Ste-Anne de Bellevue, Canada). Conjunctival scrapings from patients with tra- 
Origin of samples sequenced in this study

\begin{tabular}{lll}
\hline Identification & Year & Site \\
\hline $\mathrm{B} / 6 \cdot 71 / \mathrm{OT}$ & $1989-90$ & Child's eye \\
$\mathrm{Ba} / 6 \cdot 73 / \mathrm{OT}$ & $1989-90$ & Child's eye \\
$\mathrm{Ba} / 5 \cdot 54 / \mathrm{OT}$ & $1989-90$ & Child's eye \\
$\mathrm{Ba} / 1306 / \mathrm{OT}$ & $1989-90$ & Child's eye \\
$\mathrm{D} / 720 / \mathrm{UR}$ & 1990 & Endocervix \\
$\mathrm{D} / 1132 / \mathrm{UR}$ & 1990 & Endocervix \\
$\mathrm{Db} / 905 / \mathrm{UR}$ & 1991 & Urethra \\
$\mathrm{Db} / 2373 / \mathrm{UR}$ & 1990 & Endocervix \\
$\mathrm{Db} / 3749 / \mathrm{UR}$ & 1989 & Endocervix \\
$\mathrm{E} / 4152 / \mathrm{UR}$ & 1989 & Endocervix \\
$\mathrm{E} / 4687 / \mathrm{UR}$ & 1989 & Endocervix \\
$\mathrm{Bb} / 3969 / \mathrm{UR}$ & 1989 & Urethra \\
$\mathrm{Bb} / 4738 / \mathrm{UR}$ & 1989 & Urethra \\
$\mathrm{Bb} / 5144 / \mathrm{UR}$ & 1989 & Endocervix \\
$\mathrm{C} / 15 \cdot 164 / \mathrm{OT}$ & $1989-90$ & Child's eye \\
$\mathrm{C} / 15 \cdot 179 / \mathrm{OT}$ & $1989-90$ & Child's eye \\
$\mathrm{Ca} / 3653 / \mathrm{Ur}$ & 1990 & Urethra \\
$\mathrm{Ca} / 4471 / \mathrm{Ur}$ & 1989 & Endocervix \\
\hline ^ All samples from a child's eye came from Kenya whereas \\
endocervical and urethral samples were from the vicinity of \\
Sherbrooke, Quebec. & &
\end{tabular}

choma from 2 rural health centres (Lare and Mutuwati) in the Meru district in central Kenya, were supplied by Dr R. Brunham. ${ }^{9}$ The remaining original sample from culturepositive urogenital specimens from outpatients attending a family planning or STD referral clinic was employed to prepare DNA. ${ }^{2}$ The table identifies the samples sequenced in this study.

Primers for PCR and DNA sequencing. Primers are named by the position of the $5^{\prime}$ nucleotide in the serovar $\mathrm{C}$ sequence ${ }^{3}$ considering as position 1 the start of the leader sequence. Opposite strand primers are identified by an $R$. The nested pair of PCR primers 001, $1077 R, 076$ and $1055 R$ have already been described as primers $1,2,3$ and 4, respectively. ${ }^{2}$ Sequencing primer 371R, GCATTTGTAAACAT(C,T)TCAGCATC; primer 463, TTCAACTTAGT(T,G)GG(C, G,A)TTATTCGG; and primer 804R, AGAGGCATCCTTAGT $(T, C)$ CCTGT were also synthesised on a Pharmacia Gene Assembler by the Laboratory of Molecular Biology (Faculty of Medicine, University of Sherbrooke).

PCR procedure. PCR amplifications were carried out as described previously. ${ }^{10}$ The original clinical sample was pelleted, resuspended in $1 \mathrm{mM} \mathrm{NaOH}$ and heated for $10 \mathrm{~min}$ at $80^{\circ} \mathrm{C}$ prior to amplification with the outer pair of PCR primers (001 and $1077 \mathrm{R})$ at $1 \mu \mathrm{M}$ each in $50 \mu \mathrm{l}$ of PCR buffer (Amersham, Oakville, Ontario) containing 1.25 units of Hot Tub DNA polymerase (Amersham) and $200 \mu \mathrm{M}$ dNTP (Pharmacia, Montreal, Quebec). After an initial denaturation step for $1.5 \mathrm{~min}$ at $94^{\circ} \mathrm{C}, 39$ cycles of reannealing for 5 min at $55^{\circ} \mathrm{C}$, elongation for $3 \mathrm{~min}$ at $72^{\circ} \mathrm{C}$ and denaturation for $1 \mathrm{~min}$ at $94^{\circ} \mathrm{C}$ were performed. The final step included reannealing for $5 \mathrm{~min}$ and elongation for $10 \mathrm{~min}$. A $1 \mu \mathrm{l}$ sample of the product of this amplification reaction was subjected to an analogous series of 30 cycles of PCR using the nested primer pair 076 and 1055R. Ten $\mu \mathrm{l}$ of amplified product were analysed on $1 \%$ vertical agarose gels (100 volts, $2 \mathrm{hr}$ ) and stained with ethidium bromide.
RFLP analysis. One $\mu \mathrm{l}$ aliquots of the DNA amplified by primers 076 and $1055 R$ were digested with the restriction endonucleases AluI, MspI, HhaI, EcoRI, BclI, HinfI, HindIII, DdeI, RsaI (Pharmacia), FokI, MboI (Promega, Madison, WI), MseI, SspI, BspEI or Fnu4HI (New England Biolabs, Mississauga, Ontario) using the incubation conditions of the manufacturer. After extraction once with phenol-chloroform (1:1) the digestion products were separated on $1.5 \mathrm{~mm}$ thick, $10 \%$ polyacrylamide gels (acrylamide to bis-acrylamide ratio 19:1) in tris-borate buffer and stained with silver. ${ }^{11}$

Sequencing procedure. The DNA product amplified by nested PCR was separated on a $1 \%$ agarose gel, stained with ethidium bromide and, under long wave UV light, the approximately $970 \mathrm{bp}$ band corresponding to MOMP gene DNA was cut out of the gel. An equal volume of $0.01 \mathrm{M}$ tris $\mathrm{pH} 8.0,0.001 \mathrm{M}$ EDTA was added and the gel fragment was frozen and thawed once, squeezed and the liquid collected. A second freeze-thaw cycle was performed, the liquids recovered were pooled, extracted once with phenol and precipitated with 2 vol of ethanol. Sequencing was carried out with 40 fmoles of DNA and 1.5 pmole of oligonucleotide primer labelled with $\left[\gamma-\mathrm{P}^{32}\right]$ ATP (Amersham) by T4 polynucleotide kinase using the fmol DNA sequencing system (Promega). After addition of 5 units of sequencing grade Taq DNA polymerase and buffer, the mixture was divided into four tubes containing $2 \mu \mathrm{L}$ of $20 \mu \mathrm{M}$ each deoxyribonucleoside triphosphate with a limiting amount of a different dideoxyribonucleoside triphosphate and covered with mineral oil. After $2 \mathrm{~min}$ at $95^{\circ} \mathrm{C}$ the sequencing reaction was performed through 30 cycles of denaturation for $30 \mathrm{~s}$ at $95^{\circ} \mathrm{C}$, annealing at $60^{\circ} \mathrm{C}$ for $30 \mathrm{~s}$ and extension at $70^{\circ} \mathrm{C}$ for $1 \mathrm{~min}$. The reactions were analysed on $55 \mathrm{~cm}$ long, $8 \%$ polyacrylamide gels in $7 \mathrm{M}$ urea at 2000 volts using a Macro-phor sequencing system (LKB-Pharmacia, Montreal). Sequences were determined after autoradiography for 2 to 7 days on Hyperfilm MP (Amersham).

\section{Results}

The chlamydial MOMP gene sequences were amplified by PCR from the original clinical sample without prior growth in culture or cloning in Escherichia coli. Nested PCR provided sufficient DNA to sequence isolates present even in low amounts (less than 10 inclusion-forming units ${ }^{2}$ ).

Sequencing both strands of the MOMP gene of 14 members of the B complex from codons 15 to 86 including VSI (codons 64 to $85^{3}$ ) using primers 076 and $371 \mathrm{R}$ revealed 19 changes, 11 of which result in an amino acid substitution (see fig 1). Although most of the variation occurred in VSI, there were changes in 5 codons outside VSI that would alter the peptide sequence, including changes in codons 30 and 61 that were detectable after digestion with HhaI or NlaIII, respectively. 
Figure 1 Nucleotide and amino acid sequence comparisons of trachoma $B$ and $B a$ serovars and urogenital $B b, D, D b$ and $E$ serovars. Sequences of strains $B / T W 5 / O T$, $^{3}$ B/fali/OT (Genbank $M$ 33536), BalAp2, $D / U W-$ $3 / C x^{12}$ and $E / U W-5 / C x$ (Genbank X52557) are those published. The remaining 14 sequences were determined from specimens from ocula trachoma (identifed OT) or urogential (identified UR) sites. The unreported portion of the sequence of BalAp2 is indicated by a solid line. The boxed area represents variable segment (VS) 1 . Numbers located above codons correspond to the amino acid position in MOMP of serovar $C .^{3}$
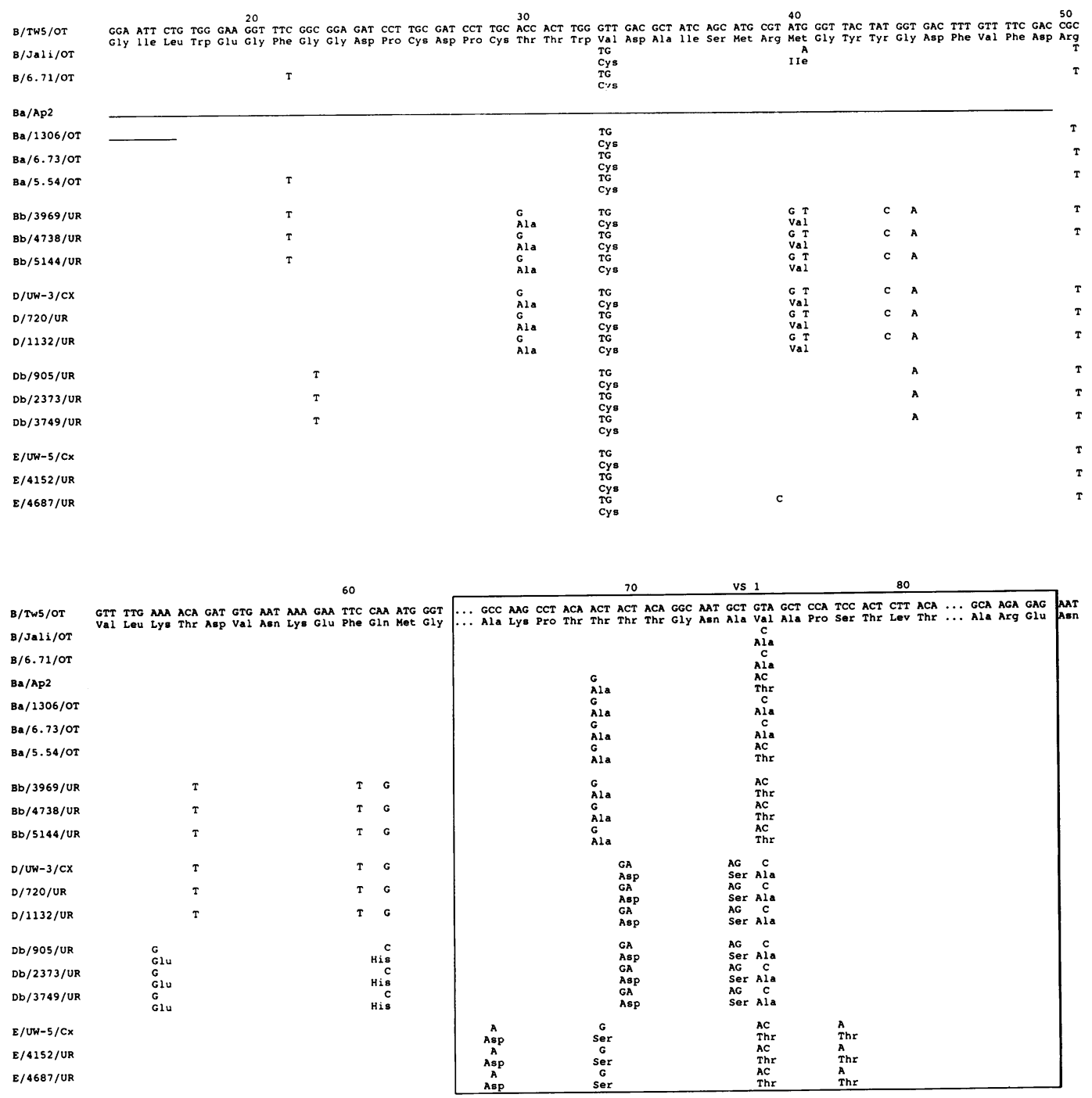

Furthermore, changes outside VSI at codons 39, 44 and 60, which do not affect the amino acid specified, affect sites cleaved by $H$ haI, $M a e I I I$ and $E c o$ RI, respectively.

The eight single-point nucleotide differences in the sequence preceding VSI between the three samples we termed $\mathrm{Db}$ and reference strain $\mathrm{D}$ would give rise to four amino acid changes (fig 1). The two changes at amino acids 30 and 40 generated sequences identical to reference strains $B$ and $E$, while the other two at amino acids 53 and 61 were observed only with $\mathrm{Db}$. Otherwise, there was no divergence of the VSI sequence between $\mathrm{D}$ and $\mathrm{Db}$. The HhaI site present in the reference $\mathrm{D}$ (codon 30 ) strain but absent in $\mathrm{Db}$ was detected in 14 of 24 serovar D samples. Also, a serovar E variant, previously observed to include an extra $H$ haI site, ${ }^{2}$ diverged only once from the reference strain sequence accounting for the observed extra HhaI site (see fig 1).

All three urogenital samples of serovar B that we term $\mathrm{Bb}$ had VSI sequences similar to the reference $\mathrm{Ba}$ strain and to $\mathrm{Ba}$ trachoma specimens (fig 1). With respect to the sequence preceding VSI, however, they differed from the trachoma samples and from reference strains of $\mathrm{Ba}$ and $\mathrm{B}$ at amino acids 30 and 40 where they mimicked serovar $D$. They also differed from $\mathrm{B}$ and $\mathrm{Ba}$ samples and resembled the $\mathrm{D}$ strain at five other nucleotides that did not affect the amino acid specified. Although variation was observed in VSI among the trachoma samples of $\mathrm{B}$ and $\mathrm{Ba}$, little variation was noted outside VSI in the three ocular trachoma $\mathrm{Ba}$ and one $\mathrm{B}$ samples that we sequenced. These results were extended to 20 other urogenital $\mathrm{Bb}$ samples that possessed an HhaI site (corresponding to amino acid 30) that was lacking in $10 \mathrm{Ba}$ and two B serovar specimens from trachoma patients.

Comparison of our sequence data between codons 109 and 239 from two trachoma samples of serovar $\mathrm{C}$ with two urogenital specimens named $\mathrm{Ca}$ revealed only one divergence in VSII from the reference strain (see fig 2). This difference could be monitored by enzyme HinfI. On the other hand, two codon changes were observed between $\mathrm{Ca}$ and $\mathrm{C}$ that would modify amino acids in the region between VSII and VSIII and a third in VSIII (that could be monitored by loss of an MseI site present in the reference serovar $C$ strain). At all four of these sites involving amino acid change the two specimens of urogenital origin (Ca) were similar to serovars $\mathrm{A}$ and $\mathrm{H}$. Four nucleotide changes that would not modify the peptide sequence also distinguished the urogenital $\mathrm{Ca}$ from the trachoma serovar $\mathrm{C}$ samples. The two serovar $\mathrm{C}$ specimens of 
Figure 2 Nucleotide and amino acid sequence comparisons of trachoma $A$ and $C$ serovars and urogenital $\mathrm{Ca}, \mathrm{H}, \mathrm{I}$ and $\mathcal{F}$ serovars. Sequences of strains $C / T W 3 / O T$, A/SA1/OT (Genbank $M 33535)$ and $H$ (Genbank X16007) are those published. Sequences C/15.164/OT,

C/15.179/OT,

Ca/3635/Ur and Cal4471/Ur were determined from specimens

from ocular trachoma

(OT) or urogenital (Ur)

sites. The unreported portions of the sequence of serovars I and $\mathcal{f}^{4}$ are indicated by a solid line. Boxed areas represent VSII and III. Numbers located above codons correspond to the amino acid position in MOMP of serovar $C$.
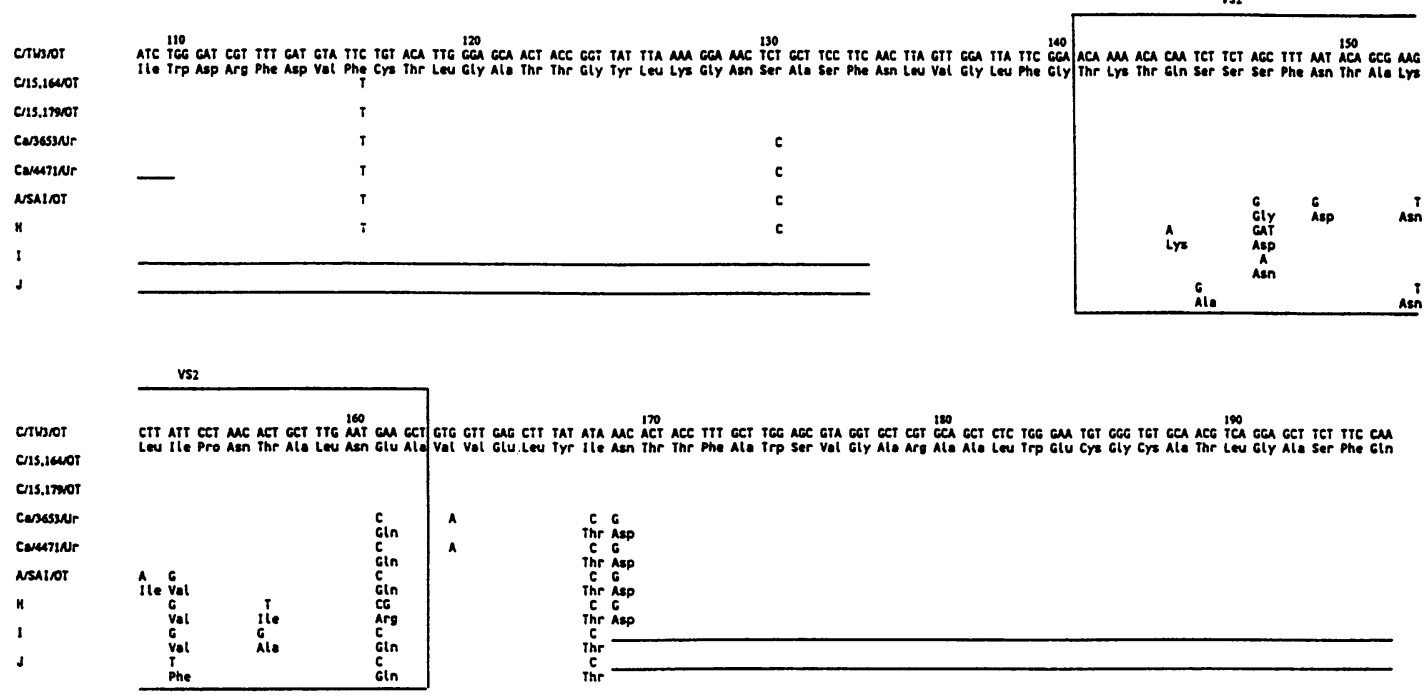

vss

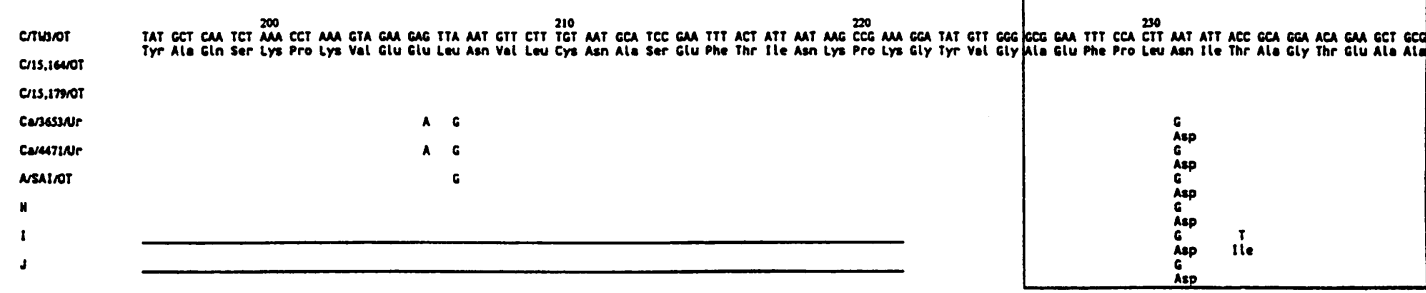

trachoma origin that were sequenced were identical to the reference $\mathrm{C}$ strain (see fig 2). The other three trachoma serovar $C$ samples from Kenya that were not sequenced and two reference strains maintained the $M s e I$ site in VSIII whereas none of seven urogenital $\mathrm{Ca}$ samples tested had the MseI site in VSIII characteristic of trachoma isolates.

Fortuitously most of the amino acid differences in VSI distinguishing $C$ complex serovars can be monitored by RFLP including an $F n u 4 \mathrm{HI}$ site unique to serovar $\mathrm{H}, F o k I$ unique to serovar $\mathrm{I}$ and $\mathrm{DdeI}$ specific to serovars A, I and Ia. ${ }^{41213}$ None of these sites were found in six urogenital $\mathrm{Ca}$ specimens tested including the two that were sequenced. One of the two amino acid modifications in VSI reported to distinguish urogenital $\mathrm{C}$ from trachoma C creates an SspI site ${ }^{7}$ which we observed in six of our Ca samples but not in four specimens from trachoma patients nor in reference $\mathrm{C}, \mathrm{J}, \mathrm{H}$ or A strains. Likewise the $M b o I$ site that could be predicted in VSIV from the published sequence of urogenital $\mathrm{C}^{7}$ or the $\mathrm{J}^{1}$ variant ${ }^{1}$ was detected in six of our $\mathrm{Ca}$ specimens but not in four trachoma C samples, 19 urogenital J specimens nor in reference $\mathrm{C}, \mathrm{J}, \mathrm{A}$ or $\mathrm{H}$ strains. The $B s p E I$ site reported in VSIV in urogenital $C$ but not in trachoma $\mathrm{C}^{7}$ was found both in six $\mathrm{Ca}$ and four of our trachoma $C$ samples as well as in reference $\mathrm{J}$ and $\mathrm{H}$, but not in reference $\mathrm{A}$ and C strains. Likewise RFLP in VSII was employed to distinguish $\mathrm{B}, \mathrm{Ba}, \mathrm{D}$ and $\mathrm{E}$. The RsaI cleavage pattern specific of $\mathrm{Ba}$ was shared by all biotype $\mathrm{Bb}$ samples $^{2} 10^{11}$ and a unique $B c l$ site in serovar $\mathrm{D}^{4}$ was also documented in all six biotype $\mathrm{Db}$ specimens tested.

\section{Discussion}

Comparison of the sequence of urogenital and trachoma specimens typed as serovar $\mathrm{Ba}$ or $\mathrm{C}$ by RFLP of amplified MOMP gene DNA indicated that although the VSI and VSII sequences were compatible with those of the reference $\mathrm{Ba}$ or $\mathrm{C}$ strains, several sequence changes outside VSI and II that would give rise to amino acid modifications distinguished the urogenital from the trachoma samples. Furthermore, urogenital isolates of serovar D could be separated into two groups on the basis of four different amino acid residues in the region of MOMP preceding VSI.

A very recent study from Winnipeg, Canada also demonstrated that urogenital $\mathrm{Ba}$ specimens were similar to trachoma $\mathrm{Ba}$ samples in VSI, II and IV. ${ }^{7}$ This same study did, however, identify differences at multiple sites in VSI, II and IV between their unique urogenital $\mathrm{C}$ sample and a reference $\mathrm{C}$ sample. Our two sequenced urogenital $\mathrm{Ca}$ specimens had identical VSII sequences to theirs whereas a urogenital sample termed $\mathrm{J}^{1}$ by another group ${ }^{1}$ had an identical VSIV to the Winnipeg group's urogenital C sample. Of the eight amino acid changes in VSI and IV distinguishing their urogenital sample from a trachoma strain, ${ }^{7}$ all six of our urogenital strains were shown by RFLP to possess at least three. The combined sequence analysis of their VSI, II and IV and our VSII, III and non-VS sequences indicated that urogenital $\mathrm{Ca}$ strains differed from reference trachoma $\mathrm{C}$ strains at 12 amino acids and at six amino acids from urogenital J strains.

Historically, serovars were first defined by different reaction patterns to antisera. The more recent use of monoclonal antibodies, 
epitope mapping and sequencing has pinpointed the differences specifying serovar allocation to VSI and VSII. ${ }^{14-16}$ VSII rather than VSI was sequenced for the $C$ complex isolates because this is the most variable of the VS in $C$ trachomatis. Indeed, VSI sequences of $\mathrm{C}$ and J serovars are identical and they differ from $\mathrm{K}$ and I serovars by only one or two amino acids, respectively, whereas there are at least four different amino acid residues in VSII between any pair of $\mathrm{C}$ complex serovars.

Variants identified by serological means or by direct sequencing, such as $\mathrm{L}_{2} \mathrm{a}, \mathrm{Da}, \mathrm{D}^{\star}$, $\mathrm{D}^{+}, \mathrm{G}^{\prime}$ and $\mathrm{I}^{\prime}{ }^{112}{ }^{13}$ have often differed by only one or two amino acid residues from the reference strain and (with the exception of Ia) been observed very rarely. Furthermore, some of them have been repeatedly passaged in culture. It would be unlikely that the genotypes $\mathrm{Ca}, \mathrm{Bb}$ and $\mathrm{Db}$ reported here would be distinguished from the reference strains by serological means as the three or four amino acid differences were observed outside the VS. They are not, however, rarely observed as we have characterised $2 \mathrm{Ca}, 3 \mathrm{Bb}$ and $3 \mathrm{Db}$ by sequencing and a further $4 \mathrm{Ca}, 17 \mathrm{Bb}$ and 7 $\mathrm{Db}$ samples could be tentatively assigned to these genotypes by identification of at least one of the nucleotide changes by RFLP. They were neither cultured nor cloned in vitro but amplified directly from the patient sample. PCR product was never reamplified with the same primers but rather further aliquots of the DNA produced with the external primers were employed to generate material with the internal primers. Thus DNA was never amplified more than once with either the external or the internal primers.

The one $\mathrm{Da}$ strain $(\mathrm{Da} / \mathrm{TW}-448$ OT) MOMP gene that has been completely sequenced ${ }^{17}$ revealed the unique amino acid change at residue 314 in VSIV that distinguished Da from D strains, ${ }^{12}{ }^{13}$ but in addition, also presented all four amino acid and nine nucleotide changes in the invariant region preceding VSI that we have shown here to distinguish $\mathrm{Db}$ from the $\mathrm{D}$ reference strain indicating that it carried both the $\mathrm{Da}$ and $\mathrm{Db}$ variations. Although it is not known whether other $\mathrm{Da}$ variants also carry the nucleotide changes typical of $\mathrm{Db}$, it is clear that the 10 $\mathrm{Db}$ samples we have analysed do not demonstrate the Da genotype as they possess the HhaI endonuclease site at amino acid 314 that is absent in Da variants. Furthermore six of 18 serovar D samples from France ${ }^{8}$ that were typed by RFLP lacked the same $H h a \mathrm{I}$ restriction site corresponding to amino acid 30 that we reported to distinguish $\mathrm{Db}$ from $\mathrm{D}$ but had not lost the HhaI site at amino acid 314 that causes $\mathrm{Da}$ to differ from $\mathrm{D}$. In contrast, the $\mathrm{Da} / \mathrm{TW}-488$ isolate lacked both this HhaI site and another in VSIV ${ }^{8}$ that distinguish all $\mathrm{Da}$ isolates from D. ${ }^{12}{ }^{13}$ This indicates that the variation observed in the relatively invariant region preceding VSI from $40 \%$ of our serovar D isolates was not a local phenomenon but was also observed in isolates from France and in one Da strain.

Urogenital $\mathrm{Bb}$ and $\mathrm{Ca}$ genotypes were con- sidered variants of serovars $B a$ and $C$ because they were most similar to $B a$ and $C$ serovars in VSI and VSII, although they resembled D or $\mathrm{H}, \mathrm{I}$ and J serovars outside VSI and VSII. It is not clear though whether $\mathrm{Bb}$ and $\mathrm{Ca}$ evolved from trachoma strains $\mathrm{Ba}$ and $\mathrm{C}$ accidentally transferred to a urogenital site or from urogenital $\mathrm{D}, \mathrm{H}, \mathrm{I}$ or J serovars. One previous study of trachoma isolates implicated genetic drift as a cause of variation within a serovar because it involved variation in only one amino acid residue per isolate. ${ }^{6}$ The variation documented here and in another report of trachoma isolates ${ }^{5}$ involved several amino acid changes without intermediate profiles in other isolates indicating that recombination between serovars may be a possible cause. The variation observed among trachoma $B$ and $\mathrm{Ba}$ isolates has usually involved regions of the gene subject to immune pressure whereas the variation reported here involved an area not suspected to be under immune pressure. The fact that in the region preceding VSI Bb coincided almost exactly with reference urogenital serovar D would imply that these are not random changes but rather indicate either a common evolutionary origin or a necessary sequence for urogenital chlamydia.

Although the effect on chlamydial biology of the variation reported here is beyond the scope of this study, it is interesting that both the $\mathrm{C}$ and $\mathrm{Ba}$ serovars found in urogenital specimens differ from those found in trachoma isolates. It is possible that this variation confers a selective advantage for multiplication in urogenital versus conjunctival epithelial cells because isolates identical to the trachoma strains are not found in urogenital specimens nor vice versa. ${ }^{9}$ It is unlikely that variation outside the immunodominant VSI could directly modify epitopes involved in serological response but it could modify their surface exposure. It has been postulated ${ }^{16}$ that enhanced surface-exposure of VS in L2 serovars contributes to their invasiveness. It will be interesting to compare the potential of $\mathrm{D}$ and $\mathrm{Db}$ to cause tubal scarring as genotype $\mathrm{Db}$ closely resembles pathogenic trachoma serovars $\mathrm{B}$ and $\mathrm{Ba}$ outside VSI whereas $\mathrm{D}$ is similar to urogenital $\mathrm{Bb}$.

We thank Robert Brunham for supplying us with samples from trachoma patients and Julie Blais, Denise Grenier, Anne Rousseau, and Carole Picard for excellent secretarial assistance.

This work was supported by Fonds FCAR grant 94ER2055.

1 Poole E, Lamont I. Chlamydia trachomatis serovar differentiation by direct sequence analysis of the variable segtiation by direct sequence analysis of the variable seg-
ment 4 region of the major outer membrane protein ment 4 region of the major outer

2 Frost EH, Deslandes S, Bourgaux-Ramoisy D. Chlamydia trachomatis serovars in 435 urogenital specimens typed by restriction endonuclease analysis of amplified DNA. $\mathscr{f}$ Infect Dis 1993;168:497-50

3 Stephens RS, Sanchez-Pescador R, Wagar EA, Inouye C Urdea MS. Diversity of Chlamydia trachomatis majo outer membrane protein genes. $f$ Bacteriol 1987 169:3879-85.

4 Yuan Y, Zhang YX, Watkins NG, Caldwell HD Nucleotide and deduced amino acid sequences for the four variable domains of the major outer membrane proteins of the 15 Chlamydia trachomatis serovars. Infect Immun 1989;57:1040-9. 
5 Dean D, Schachter J, Dawson CR, Stephens RS. Comparison of the major outer membrane protein variant sequence regions of $\mathrm{B} / \mathrm{Ba}$ isolates: a molecular epidemiologic approach to Chlamydia trachomatis infections. miologic approach to Chlamydia

6 Hayes LJ, Bailey RL, Mabey DCW, et al. Genotyping of Chlamydia trachomatis from a trachoma-endemic village in the Gambia by a nested polymerase chain reaction identification of strain variants. $\mathcal{F}$ Infect Dis 1992;166 1173-7.

7 Yang CL, Maclean I, Brunham RC. DNA sequence polymorphism of the Chlamydia trachomatis omp 1 gene. $\mathcal{F}$ Infect Dis 1993;168:1225-30.

8 Rodriguez P, de Barbeyrac B, Persson K, Dutilh B, Bebear C. Evaluation of molecular typing for epidemiological C. Evaluation of molecular typing for epidemiological study of Chlamydia trachomatis gen

9 Datta $P$, Frost E, Peeling R, et al. Ophthalmia neonatorum in a trachoma endemic area. Sex Transm Dis 1994, 21:1-4

10 Frost EH, Deslandes S, Bourgaux-Ramoisy D. Sensitive detection and typing of Chlamydia trachomatis using nested polymerase chain reaction. Genitourin Med 1993;69:290-4.

11 Frost EH, Deslandes S, Veilleux S, Bourgaux-Ramoisy D. Typing Chlamydia trachomatis by detection of restriction fragment length polymorphism in the gene encoding the major outer membrane protein. I Infect Dis 1991;163: 1103-7.

12 Dean $D$, Patton $M$, Stephens RS. Direct sequence evaluation of the major outer membrane protein gene variant regions of Chlamydia trachomatis subtypes $\mathrm{D}^{\prime}, \mathrm{I}^{\prime}$ and $\mathrm{L}_{2}^{\prime}$. regions of Chlamydia trachomatis

13 Lampe MF, Suchland RJ, Stamm WE. Nucleotide sequence of the variable domains within the major oute membrane protein gene from serovariants of Chlamydia trachomatis. Infect Immun 1993;61:213-9.

14 Baehr W, Zhang XY, Joseph T, et al. Mapping antigenic domains expressed by Chlamydia trachomatis major outer membrane protein genes. Proc Natl Acad Sci USA 1988;85:4000-4.

15 Stephens RS, Wagar EA, Schoolnik GK. High-resolution mapping of serovar-specific and common antigenic determinants of the major outer membrane protein
Chlamydia trachomatis. $\mathcal{E}$ Exp Med 1988;167:817-31.

16 Zhong G, Brunham RC. Antigenic determinants of the chlamydial major outer membrane protein resolved at single amino acid level. Infect Immun 1991;59:1141-7.

17 Sayada C, Denamur E, Elion J. Complete sequence of the major outer membrane protein-encoding gene of Chlamydia trachomatis serovar Da. Gene 1992;120: 129-30. 